\title{
Nonlinear optical susceptibility of multicomponent tellurite thin film glasses
}

\author{
D. Munoz-Martin, ${ }^{1, a)}$ H. Fernandez, ${ }^{1}$ J. M. Fernandez-Navarro, ${ }^{1}$ J. Gonzalo, ${ }^{1}$ J. Solis, ${ }^{1}$ \\ J. L. G. Fierro, ${ }^{2}$ C. Domingo, ${ }^{3}$ and J. V. Garcia-Ramos ${ }^{3}$ \\ ${ }^{1}$ Laser Processing Group, Instituto de Optica (CSIC), Serrano 121, 28006 Madrid, Spain \\ ${ }^{2}$ Instituto de Catálisis y Petroleoquímica (CSIC), Marie Curie s/n, 28049 Cantoblanco, Spain \\ ${ }^{3}$ Instituto de Estructura de la Materia (CSIC), Serrano 121, 28006 Madrid, Spain
}

(Received 6 June 2008; accepted 29 September 2008; published online 2 December 2008)

\begin{abstract}
Tellurite $\left(\mathrm{TeO}_{2}-\mathrm{TiO}_{2}-\mathrm{Nb}_{2} \mathrm{O}_{5}\right)$ thin film glasses have been produced by pulsed laser deposition. The dispersion of the real and imaginary parts of the linear refractive index has been measured in the range from 300 to $1700 \mathrm{~nm}$. Films present high refractive index $(n=2.01)$ and reduced absorption $\left(k<10^{-4}\right)$ at $\lambda=1500 \mathrm{~nm}$. The nonlinear third order optical susceptibility $\left(\left|\chi^{(3)}\right|\right)$ has been determined at four different wavelengths $(600,800,1200$, and $1500 \mathrm{~nm})$. The out-of-resonance $\left|\chi^{(3)}\right|$ values $\left(\sim 10^{-12} \mathrm{esu}\right)$ are found to be ten times higher than those of the bulk glass and $10^{2}$ times higher than that of silica. Compositional and structural analysis reveals an increase of both the Ti atomic content and the fraction of nonbridging oxygen bonds in the deposited films. Both factors lead to a higher hyperpolarizability of the film constituents that is proposed to be responsible for the high $\left|\chi^{(3)}\right|$ value of the films. (C) 2008 American Institute of Physics. [DOI: 10.1063/1.3021052]
\end{abstract}

\section{INTRODUCTION}

Glasses are promising media for the development of photonic devices, which are the basic elements for the advance of optical communication networks and optical computing. ${ }^{1-3}$ In particular, $\mathrm{TeO}_{2}$ based glasses have been the subject of a great research effort during the past decade. They are transparent in the visible and near IR regions (from $400 \mathrm{~nm}$ to $6 \mu \mathrm{m})$ and show a high refractive index $(n \geq 2)$, which allows the fabrication of fibers and waveguides with strong light confinement. ${ }^{4}$ Beside these remarkable properties, they stand out for their large nonlinear optical response, as they show some of the highest values of nonlinear refractive index among oxide glasses, which are 10-100 times larger than that of fused silica. ${ }^{3-8}$ These high values are essential for the development of active nonlinear photonic devices, such as all-optical switches or modulators. ${ }^{1,2,5,6}$

However, the successful application of these materials depends not only on their excellent optical properties but also on the capability of producing them in functional configurations such as fibers or thin films. Commercial fiber amplifiers made of tellurite glasses are already available, ${ }^{4}$ but there have been only a few attempts to produce high optical quality tellurite thin film glasses by sol-gel processing, ${ }^{9}$ sputtering ${ }^{10}$ or pulsed laser deposition (PLD) ${ }^{11}$ PLD has proven to be an excellent technique to produce complex oxide films ${ }^{12}$ and, in particular, it has been used to produce heavy metal oxide thin film glasses in a wide range of compositions with good optical properties. ${ }^{13,14}$ This work focuses on the deposition of complex transparent tellurite thin film glasses by PLD with large nonlinear refractive index. The linear and nonlinear optical responses of the deposited films

a)Electronic mail: david@io.cfmac.csic.es. are compared to those of bulk glass and the observed differences are discussed in terms of the compositional and structural particularities of the films.

\section{EXPERIMENTAL}

$\mathrm{TeO}_{2}$-based thin film glasses were produced by PLD using an ArF excimer laser $[\lambda=193 \mathrm{~nm}, \tau=20 \mathrm{~ns}$ full width at half maximum (FWHM)]. The laser was focused on the surface of a glass target to lead to an energy density of $1.5 \mathrm{~J} \mathrm{~cm}^{-2}$. The deposition process took place in a vacuum chamber first evacuated to a residual pressure of 7 $\times 10^{-4} \mathrm{~Pa}$ and then filled with oxygen up to a dynamic pressure of $10 \mathrm{~Pa}$. Films with a thickness of $3 \mu \mathrm{m}$ were deposited on Corning glass substrates held at room temperature.

The target was a bulk glass with a nominal composition of $90 \mathrm{TeO}_{2} \cdot 5 \mathrm{TiO}_{2} \cdot 5 \mathrm{Nb}_{2} \mathrm{O}_{5}(\mathrm{~mol} \%)$. This glass was obtained using standard melting methods from high purity reagents: $\mathrm{TeO}_{2}$ (Alfa 99.99\%), $\mathrm{TiO}_{2}$ (Sigma Aldrich 99.99\%), and $\mathrm{Nb}_{2} \mathrm{O}_{5}$ (Alfa 99.9985\%). The mixture was melted in a platinum crucible at a temperature of $740{ }^{\circ} \mathrm{C}$ for $45 \mathrm{~min}$, then heated up to $760{ }^{\circ} \mathrm{C}$ for $5 \mathrm{~min}$ and finally poured onto a preheated brass plate, annealed $15 \mathrm{~min}$ at $400{ }^{\circ} \mathrm{C}$ and cooled at $3{ }^{\circ} \mathrm{C} \mathrm{min}^{-1}$ down to room temperature ${ }^{15}$

The composition and oxidation state of the cations present in the powder reagents and the bulk and film glasses were measured by $\mathrm{x}$-ray photoelectron spectroscopy (XPS). XP spectra were recorded on a spectrometer equipped with a hemispherical electron analyzer and a $\operatorname{Mg} K \alpha \quad(h \nu$ $=1253.6 \mathrm{eV}) \mathrm{x}$-ray source $(12 \mathrm{kV}$ and $10 \mathrm{~mA})$. The base pressure of the analysis chamber was maintained below 3 $\times 10^{-7} \mathrm{~Pa}$ during data acquisition. Peak intensities were estimated by calculating the integral of each peak after smoothing and subtracting a Shirley-type background as described in Ref. 16. Binding energies were referenced to the $\mathrm{C} 1 s$ line at $284.9 \mathrm{eV}$. Depth profiling was achieved by $\mathrm{Ar}^{+}$sputtering 
(1.7 $\mathrm{kV}$, sputtering rate $\sim 1 \mathrm{~nm} \mathrm{~min}^{-1}$ ), and three independent measurements were made in each sample. The absolute errors in the quantitative XPS measurements are estimated to be $\pm 5 \%$ in the cases of $\mathrm{Te}$ and $\mathrm{O}$ and $\pm 20 \%$ in that of $\mathrm{Ti}$ and Nb. ${ }^{13}$ The glass network structure was analyzed by Raman spectroscopy. Raman spectra were recorded with a confocal Raman microscope equipped with a diode laser emitting at $785 \mathrm{~nm}$. The output laser power was $30 \mathrm{~mW}$ and the beam was focused on the sample using a $50 \times$ objective. The elastic scattering was eliminated using a notch filter and the signal was detected through an electrically refrigerated chargecoupled device camera. Acquisition time was $10 \mathrm{~min}$.

The linear and nonlinear optical properties of both bulk and film glasses have been characterized by spectroscopic ellipsometry and degenerate four wave mixing (DFWM) respectively. The experimental data measured by ellipsometry in the range from 300 to $1700 \mathrm{~nm}$ were fitted using a Cauchy-type dispersion for the real part of the linear refractive index $(n)$ and an exponential decay for the imaginary part $(k)$,

$$
\begin{aligned}
& n(\lambda)=A_{n}+\frac{B_{n}}{\lambda^{2}}+\frac{C_{n}}{\lambda^{4}}, \\
& k(\lambda)=A_{k} \exp \left[B_{k}\left(\frac{1}{\lambda}-\frac{1}{\gamma}\right)\right],
\end{aligned}
$$

where $A_{n}, B_{n}, C_{n}, A_{k}$, and $B_{k}$ are adjustable parameters and $\gamma$ is the optical gap expressed in nanometers. The absorption spectra of a $1 \mathrm{~mm}$ thick bulk glass and the film glass samples were also measured in the range from 300 to $700 \mathrm{~nm}$ using a spectrophotometer and an ellipsometer, respectively. Finally, the modulus of the diagonal component of the third order optical susceptibility tensor $\left(\left|\chi_{x x x x}^{(3)}\right|\right)$ was measured by DFWM in the forward folded box configuration. ${ }^{17}$ Two different excitation sources were used to measure $\left|\chi_{x x x x}^{(3)}\right|$ at four different wavelengths $(600,800,1200$, and $1500 \mathrm{~nm})$. The first of them was a $1 \mathrm{kHz}$ repetition rate femtosecond Ti:sapphire regenerative amplifier operating at $\lambda=800 \mathrm{~nm}$. The pulse compressor of the amplifier was adjusted to precompensate the dispersion caused by the optical elements located in the beam path in order to produce the shortest possible pulse $(\approx 100 \mathrm{fs}$ ) at the sample. The second source was an optical parametric amplifier pumped with the regenerative amplifier output and configured to operate at the three other indicated wavelengths $(600,1200$, and $1500 \mathrm{~nm})$. In all cases, the excitation laser beam was split in three parallel arms with equal power, allowing separate control of the beam polarization and the relative delay between pulses. The beams were overlapped at the sample by means of a $75 \mathrm{~mm}$ focal length lens leading to a beam waist of $\approx 40 \mu \mathrm{m}$. The intensity of the conjugated beam followed a characteristic cubic dependence as a function of the pump beam intensity, whereas the use of parallel polarizations for all the beams allowed accessing $\left|\chi_{x x x x}^{(3)}\right|$ that we will refer from now as $\left|\chi^{(3)}\right|$. The absolute value of $\left|\chi^{(3)}\right|$ was finally evaluated by using a fused silica plate as reference material $\left(\left|\chi^{(3)}\right|_{\mathrm{SiO} 2}\right.$ $\left.=1.5 \pm 0.5 \times 10^{-14} \mathrm{esu}\right)^{17}$
TABLE I. Composition of bulk and film glasses in atomic percentage. The table includes the value of $n$ at $590 \mathrm{~nm}$ (visible) and $1500 \mathrm{~nm}$ (IR). $\Delta n$ is \pm 0.01 in all cases

\begin{tabular}{lcccccc}
\hline \hline & \multicolumn{5}{c}{$\begin{array}{c}\text { Composition } \\
(\text { at. \% })\end{array}$} \\
\cline { 2 - 7 } Sample & $\mathrm{Te}$ & $\mathrm{Ti}$ & $\mathrm{Nb}$ & $\mathrm{O}$ & $590 \mathrm{~nm}$ & $1500 \mathrm{~nm}$ \\
\hline Bulk & $27 \pm 1.4$ & $1.8 \pm 0.4$ & $3.2 \pm 0.6$ & $68 \pm 3$ & 2.20 & 2.12 \\
Film & $25 \pm 1.3$ & $3.3 \pm 0.6$ & $4.0 \pm 0.8$ & $67 \pm 3$ & 2.07 & 2.01 \\
\hline \hline
\end{tabular}

In the case of film glasses, the contributions of the substrate to the Raman and absorption spectra and to the DFWM measurements were carefully subtracted.

\section{RESULTS}

Table I shows the composition of bulk and film glasses in atomic percentages determined by XPS. The content of the main elements ( $\mathrm{Te}$ and $\mathrm{O}$ ) shows small differences that are within the experimental error. However, in the case of the cations acting as glass modifiers ( $\mathrm{Ti}$ and $\mathrm{Nb}$ ), films show a moderate increase $(25 \%)$ and a strong enhancement $(80 \%)$ in the relative contents of $\mathrm{Nb}$ and $\mathrm{Ti}$, respectively, when compared to the bulk glass. XPS analysis shows no significant changes in the binding energies of the different elements. Therefore, the oxidation states of the cations should be the same than in the original powder reagents $\left(\mathrm{Te}^{4+}, \mathrm{Ti}^{4+}\right.$, and $\mathrm{Nb}^{5+}$ ) and there is no oxidation or reduction in the cations after the synthesis of the bulk or film glasses.

The reduced Raman spectra ${ }^{18}$ of bulk and film glasses are shown in Fig. 1. In the case of the bulk glass, the spectrum is similar to that reported previously for pure $\mathrm{TeO}_{2}$ glass ${ }^{19}$ and multicomponent tellurite glasses with low content of network modifiers. ${ }^{20-22}$ The spectrum has been normalized to the intensity of the peak at $660 \mathrm{~cm}^{-1}$ and it clearly shows three different bands, indicated in the figure as $A, B$, and $C$. The $A$ band at $400-500 \mathrm{~cm}^{-1}$ and $B$ band at $660 \mathrm{~cm}^{-1}$ are associated to the vibration of symmetric and antisymmetric $\mathrm{Te}-\mathrm{O}-\mathrm{Te}$ linkages, respectively, related to a continuous network built up by $\left[\mathrm{TeO}_{4}\right]$ structural units, called trigonal bypyramids. Finally, the $C$ band, at frequencies between 700 and $800 \mathrm{~cm}^{-1}$, is related to the presence of

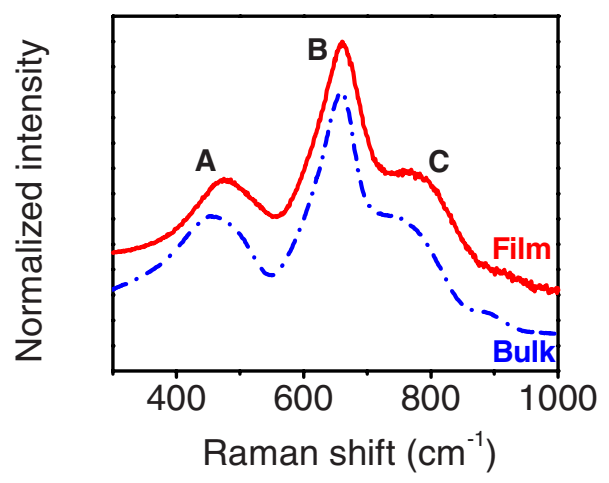

FIG. 1. (Color online) Raman spectra of (dashed-dotted line) bulk and (solid line) film glasses. The intensity is normalized to that of the main peak at $660 \mathrm{~cm}^{-1}$. The bands are named as $A, B$, and $C$. Raman spectra have been shifted vertically to ease comparison 


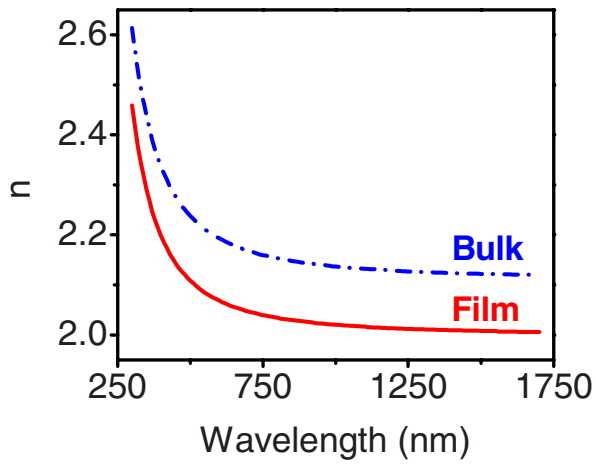

FIG. 2. (Color online) Dispersion of the refractive index of (dashed-dotted line) bulk and (solid line) film glasses obtained from the ellipsometrical data.

nonbridging oxygens (NBO) associated to structural units different than $\left[\mathrm{TeO}_{4}\right]:\left[\mathrm{TeO}_{3+1}\right]$ polyhedrons and $\left[\mathrm{TeO}_{3}\right]$ trigonal pyramids. ${ }^{4,19-22}$ Besides those peaks, the small band observed at $900 \mathrm{~cm}^{-1}$ is related to the presence of $\left[\mathrm{NbO}_{6}\right]$ structural units. ${ }^{23,24}$ The same bands are clearly identified in the case of films. The only relevant differences between the two spectra are in the $A$ and $C$ bands, which are shifted toward higher frequencies in the case of films. The relative intensity of the $A$ band is slightly lower than in the case of bulk glass, while the $C$ band shows the opposite behavior.

Figure 2 shows the dispersion of $n$ in the studied spectral range both for bulk and thin film glass samples. It shows a strong decay for wavelengths up to $600 \mathrm{~nm}$ and a slow decrease at longer wavelengths. The values measured for films are a 5\% lower than those measured for the bulk glass in all the spectral range studied. Values of $n$ at 590 and $1500 \mathrm{~nm}$ have been also included in Table I. Figure 3 shows the absorption spectra of bulk and film glasses. They show a sharp absorption edge that is apparently blueshifted in the case of the films with respect to that of the bulk. Defining the absorption edge as the wavelength at which the absorbance is $0.5,{ }^{15}$ the edges are observed to occur at $\lambda \approx 440 \mathrm{~nm}$ and $\lambda$ $\approx 390 \mathrm{~nm}$, which corresponds to optical gaps of $E_{B}$ $\approx 2.82 \mathrm{eV}$ and $E_{F} \approx 3.18 \mathrm{eV}$, for bulk and film glasses, respectively. Bulk and film glasses have low absorption for wavelengths longer than their respective absorption edges. The inset in Fig. 3 shows that the imaginary part of the

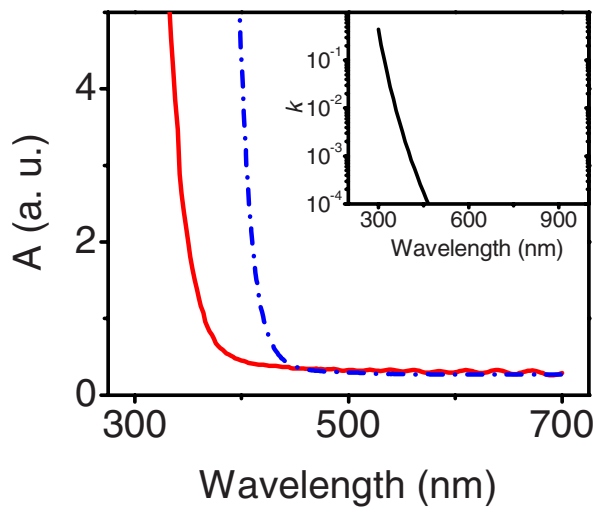

FIG. 3. (Color online) Absorption spectra of (dashed-dotted line) bulk and (solid line) film glasses. The inset shows the imaginary part of the refractive index of the films obtained from the ellipsometric data.

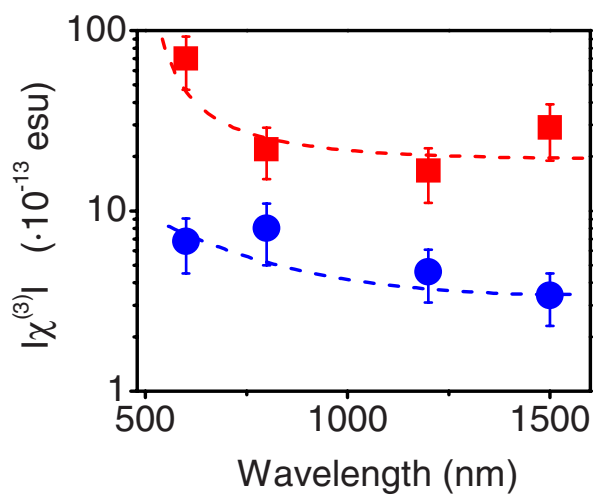

FIG. 4. (Color online) Wavelength dependence of $\left|\chi^{(3)}\right|$ for (๑) bulk and (घ) film glasses. Dashed lines are guides for the eye.

refractive index $(k)$ of the films is below our experimental resolution limit $\left(<10^{-4}\right)$ for wavelengths longer than 450 $\mathrm{nm}$.

The nonlinear optical susceptibility of bulk $\left(\left|\chi^{(3)}\right|_{B}\right)$ and film $\left(\left|\chi^{(3)}\right|_{F}\right)$ glasses is shown in Fig. 4 for the four wavelengths measured. At $1500 \mathrm{~nm},\left|\chi^{(3)}\right|_{B}$ is $3 \times 10^{-13}$ esu, which is one order of magnitude larger than that of silica, while $\left|\chi^{(3)}\right|_{F}$ is one order of magnitude larger than that of bulk glass $\left(3 \times 10^{-12}\right.$ esu). $\left|\chi^{(3)}\right|$ remains approximately constant in both cases for wavelengths in the range from 1500 to $1200 \mathrm{~nm}$ to show a moderate increase at shorter wavelengths $\left(\left|\chi^{(3)}\right|_{B}=7 \times 10^{-13}\right.$ esu and $\left|\chi^{(3)}\right|_{F}=7 \times 10^{-12}$ esu at $\left.600 \mathrm{~nm}\right)$.

\section{DISCUSSION}

The optical behavior of tellurite film glasses shows two significant differences when compared to that of bulk glass: first, films have a 5\% smaller refractive index and second, $\left|\chi^{(3)}\right|$ is one order of magnitude higher in the films than in the bulk glass. The value of $n$ measured for the bulk glass is in good agreement with those previously reported for $\mathrm{TeO}_{2}-\mathrm{TiO}_{2}$ and $\mathrm{TeO}_{2}-\mathrm{Nb}_{2} \mathrm{O}_{5}$ binary glasses ${ }^{8,25}$ and other glasses of the ternary system $\mathrm{TeO}_{2}-\mathrm{TiO}_{2}-\mathrm{Nb}_{2} \mathrm{O}_{5}$. ${ }^{7}$ The smaller value of $n$ in the case of film glasses could be related to the compositional variations observed in the films (Table I), which consist mainly in an enrichment in Ti. Since XPS experiments have shown no changes in the oxidation state of the cations in the films with respect to the bulk glass, films must be a mixture of the original oxides. Therefore, the increase in $\mathrm{Ti}$ atomic content enhances the content of $\mathrm{TiO}_{2}$ with respect to the bulk glass. Previous results in bulk $\mathrm{TeO}_{2}-\mathrm{TiO}_{2}$ glasses have shown that the increase in the $\mathrm{TiO}_{2}$ content contributes to increase the linear and nonlinear refractive index of glasses due to the large polarizability of the empty $d$-orbital in $\mathrm{Ti}^{4+} \cdot{ }^{22,25-28}$ However, $n$ decreases in the case of films as shown in Fig. 2. In order to understand this behavior, we must take into account that $n$ is related not only to the polarizability of their constituents but also to their density $(\rho)$ in the case of optical materials, ${ }^{29}$

$$
n=\sqrt{\frac{1+A \rho}{1-A \rho}}, \text { with } A=\left(\frac{4 \pi}{3}\right) \frac{\alpha_{m} N_{A}}{M},
$$

where $\alpha_{m}$ and $M$ are the average polarizability and molecular weight of the compound, respectively, and $N_{A}$ is Avogadro's 
TABLE II. Atomic composition and $\left|\chi^{(3)}\right|$ values at $1500 \mathrm{~nm}$ for the three different bulk glasses and the film deposited from the glass used as target (glass I). In the case of glass I and the film the experimental compositions measured by XPS are shown, while in the case of the other two bulk glasses the atomic composition is obtained from the nominal molar composition.

\begin{tabular}{|c|c|c|c|c|c|}
\hline \multirow[b]{2}{*}{ Sample } & \multicolumn{4}{|c|}{$\begin{array}{l}\text { Composition } \\
\text { (at. \%) }\end{array}$} & \multirow{2}{*}{$\begin{array}{l}\left|\chi^{(3)}\right| \text { at } 1.5 \mu \mathrm{m} \\
\left(\times 10^{-13} \text { esu }\right)\end{array}$} \\
\hline & $\mathrm{Te}$ & $\mathrm{Ti}$ & $\mathrm{Nb}$ & $\mathrm{O}$ & \\
\hline Glass I $90 \mathrm{TeO}_{2}-5 \mathrm{TiO}_{2}-5 \mathrm{Nb}_{2} \mathrm{O}_{5}$ & $27 \pm 1.4$ & $1.8 \pm 0.4$ & $3.2 \pm 0.6$ & $68 \pm 3$ & $3 \pm 1$ \\
\hline Glass II $85 \mathrm{TeO}_{2}-10 \mathrm{TiO}_{2}-5 \mathrm{Nb}_{2} \mathrm{O}_{5}$ & 26.6 & 3.1 & 3.1 & 67.2 & $5 \pm 2$ \\
\hline Glass III $80 \mathrm{TeO}_{2}-10 \mathrm{TiO}_{2}-10 \mathrm{Nb}_{2} \mathrm{O}_{5}$ & 23.5 & 3.0 & 5.9 & 67.6 & $6 \pm 2$ \\
\hline Film glass & $25 \pm 1.3$ & $3.3 \pm 0.6$ & $4.0 \pm 0.8$ & $68 \pm 3$ & $30 \pm 10$ \\
\hline
\end{tabular}

number. Thin films have been found to have a lower density than bulk materials due to a higher porosity degree ${ }^{30}$ which leads to a reduced optical density and $n$. To evaluate this possibility, the ellipsometric data of the films have been simulated using the Bruggeman effective medium. ${ }^{31}$ This model predicts the optical properties of a microstructural random mixture of two different compounds, weighted by their volume fractions. Assuming that these compounds are the bulk material and an unknown fraction of voids representing the porosity of the films, the optical properties of films can be well simulated in our case considering a volume fraction of voids of $7.5 \%$. This suggests that tellurite films have densities $7.5 \%$ lower than bulk samples. These results are in good agreement with the values of the refractive index predicted by Eq. (3) and with previous experimental measurements of the density of heavy metal oxide thin films grown by PLD in an oxygen atmosphere. ${ }^{14}$

In the case of the nonlinear optical response, $\left|\chi^{(3)}\right|_{F}$ is one order of magnitude larger than $\left|\chi^{(3)}\right|_{B}$, but both bulk and film glasses show a similar spectral dependence of $\left|\chi^{(3)}\right|$ (Fig. 4). The values of $\left|\chi^{(3)}\right|_{B}$ and $\left|\chi^{(3)}\right|_{F}$ measured at $600 \mathrm{~nm}$ (and also at $800 \mathrm{~nm}$ in the case of bulk glasses) show a moderate increase with respect to those determined at 1200 and 1500 $\mathrm{nm}$. The corresponding photon energies $\left(E_{\mathrm{ph}}\right)$ for the four wavelengths considered are $2.07 \mathrm{eV}(600 \mathrm{~nm}), 1.55 \mathrm{eV}(800$ $\mathrm{nm}), 1.03 \mathrm{eV}(1200 \mathrm{~nm})$, and $0.83 \mathrm{eV}(1500 \mathrm{~nm})$, thus the condition for two photon absorption (TPA) $\left(2 E_{\mathrm{ph}} \geq E_{B}, E_{F}\right)$ is satisfied at 600 and $800 \mathrm{~nm}$ in the case of bulk glasses, and at $600 \mathrm{~nm}$ in the case of film glasses. ${ }^{5}$ TPA contributes to the imaginary part of $\chi^{(3)}\left[\operatorname{Im}\left(\chi^{(3)}\right)\right]$ that leads to the observed moderate increase in $\left|\chi^{(3)}\right|$ at the above mentioned wavelengths. ${ }^{1}$ On the contrary, $\operatorname{Im}\left(\chi^{(3)}\right)$ should be negligible at 1200 and $1500 \mathrm{~nm}$, and thus only the real part of $\chi^{(3)}$ $\left[\operatorname{Re}\left(\chi^{(3)}\right)\right]$ contributes to $\left|\chi^{(3)}\right|$. Therefore, the values measured at these wavelengths are essentially of nonresonant character and lower than those measured at the short wavelengths where TPA contributes to $\left|\chi^{(3)}\right|$, as experimentally observed.

The magnitude of the third order nonlinear response of the bulk glass at $1500 \mathrm{~nm}$ is comparable to those reported in literature for $\mathrm{TeO}_{2}$ based glasses measured using other techniques such as Z-scan $\left(n_{2}=3.8 \times 10^{-19} \mathrm{~m}^{2} \mathrm{~W}^{-1}\right)$ (Ref. 6) or time resolved interferometry $\left(n_{2}=6-7 \times 10^{-19} \mathrm{~m}^{2} \mathrm{~W}^{-1}\right) .{ }^{8}$ To perform this comparison we have taken into account that $\left|\chi^{(3)}\right|_{B} \approx \operatorname{Re}\left(\chi_{B}^{(3)}\right)$ at $1500 \mathrm{~nm}$, and we have evaluated $n_{2}$ $\propto \operatorname{Re}\left(\chi^{(3)}\right)$ using the procedure described in Ref. 17. This leads to $n_{2}=2.5 \pm 0.8 \times 10^{-19} \mathrm{~m}^{2} \mathrm{~W}^{-1}$. Semiempirical models for the nonlinear response of glasses ${ }^{1,3,5}$ predict that higher $n$ values lead to higher nonlinear susceptibilities. However, tellurite films glasses produced in the present work show a slightly lower $n$, while having higher $\left|\chi^{(3)}\right|$ when compared to bulk tellurites. Thus, this enhancement must be related to the compositional and structural differences observed in the films with respect to the bulk glass.

In the first place, the Ti enrichment observed in the films may contribute to that enhancement since the presence of $\mathrm{TiO}_{2}$ has been shown to enhance the nonlinear response by a $10 \%$ in $\mathrm{TiO}_{2}-\mathrm{TeO}_{2}$ binary glasses. ${ }^{25}$ In particular, an increase in $\left|\chi^{(3)}\right|$ by a factor of 3 has been reported in silicabased glasses when increasing the $\mathrm{TiO}_{2}$ content from 10 to $30 \mathrm{~mol} \%{ }^{27}$ Moreover, the increase in the relative content of different glass network modifiers contributes to increase the concentration of NBO, which have high hyperpolarizability. ${ }^{32}$ We have analyzed this possibility by preparing additional bulk glasses with $\mathrm{TiO}_{2}$ contents similar to that of the film glasses and measuring their nonlinear response. Table II summarizes the compositions and $\left|\chi^{(3)}\right|$ values at $1500 \mathrm{~nm}$ for the bulk glass used as target (glass I), the additional bulk glasses $\left(85 \mathrm{TeO}_{2} \cdot 10 \mathrm{TiO}_{2} \cdot 5 \mathrm{Nb}_{2} \mathrm{O}_{5}\right.$, glass II, and $80 \mathrm{TeO}_{2} \cdot 10 \mathrm{TiO}_{2} \cdot 10 \mathrm{Nb}_{2} \mathrm{O}_{5}$, glass III), and the deposited film glasses. $\left|\chi^{(3)}\right|$ increases only by a factor of 2 when increasing the Ti content in bulk glasses, which suggests the existence of additional processes contributing to the strong enhancement of $\left|\chi^{(3)}\right|$ observed in the films.

The exact origin of the nonlinearity of heavy metal oxide glasses is still object of active research. In the case of tellurite glasses, the high hyperpolarizability of $\mathrm{TeO}_{2}$ has been attributed to the empty $5 d$ orbitals $^{26}$ or to the electron lone pair at the Te atom. ${ }^{28}$ XPS analysis confirms no changes in the Te atomic content or in the oxidation state of Te and thus, we do not expect any additional contribution to $\left|\chi^{(3)}\right|$ from these factors. Recent $a b$ initio calculations ${ }^{33}$ have related the hyperpolarizability of $\mathrm{TeO}_{2}$ glass to electron delocalization in $\left(\mathrm{TeO}_{2}\right)_{n}$ chainlike structures, which suggests that the structural differences observed in the film glasses may be responsible of the enhancement of the nonlinear response. We have analyzed their potential effect on the optical nonlinear response of film glasses by annealing them in air at $T=350{ }^{\circ} \mathrm{C}$ for $90 \mathrm{~min}$. This should relax the glass film structure making it similar to that of bulk glasses. The $\left|\chi^{(3)}\right|$ value 
at $1500 \mathrm{~nm}$ for the annealed film glasses decreases down to $3 \pm 2 \times 10^{-13} \mathrm{esu}$, which is similar to $\left|\chi^{(3)}\right|_{B}$ and thus confirms the role of the film glass structure on the observed increase in $\left|\chi^{(3)}\right|_{F}$.

Raman analysis of the films shows a lower intensity of the band at $400-500 \mathrm{~cm}^{-1}$ ( $A$ band) and a higher intensity of the band at $700-800 \mathrm{~cm}^{-1}$ ( $C$ band) when compared to the spectrum of the bulk glass (Fig. 1). These differences are similar to those reported between liquid $\mathrm{TeO}_{2}$ and $\mathrm{TeO}_{2}$ glass, ${ }^{34}$ although less obvious in our case. They suggest that the glass network of the films is made by shorter $\mathrm{TeO}_{2}$ chains with a higher concentration of terminal $\mathrm{Te}-\mathrm{O}$ bonds than in the case of bulk glass. According to Ref. 33 these structural characteristics have opposite effects on the nonlinear response: the slightly shorter length of $\mathrm{TeO}_{2}$ chains in the films should reduce their $\left|\chi^{(3)}\right|$, while the increase in the concentration of terminal $\mathrm{Te}-\mathrm{O}$ bonds contributes to a larger concentration of NBO and thus, to an increase in $\left|\chi^{(3)}\right|$. From the results presented in this work, the second contribution seems to be dominant and thus we conclude that the enhancement of $\left|\chi^{(3)}\right|$ observed in tellurite film glasses with respect to the values measured in the starting bulk glass is related to both the increase in $\mathrm{TiO}_{2}$ content in the films and the creation of NBO, without the addition of network modifiers due to the more disordered glass state in the films that is associated to the intrinsic characteristics of the PLD process.

\section{CONCLUSIONS}

Multicomponent $\mathrm{TeO}_{2}$-based thin film glasses have been produced by PLD. Film glasses have excellent optical properties, including high refractive index $(2.01$ at $1500 \mathrm{~nm})$, high transmission for wavelengths longer than $450 \mathrm{~nm}$, and large $\left|\chi^{(3)}\right|$ value $\left(3 \times 10^{-12}\right.$ esu at $\left.1500 \mathrm{~nm}\right)$ that are one order of magnitude higher than that of the starting bulk glass and two orders of magnitude higher than that of the $\mathrm{SiO}_{2}$ glass.

The optical differences between film and bulk glasses have been discussed in terms of the composition and the structure of the respective glass networks. The decrease in the refractive index in the films with respect to the bulk glass suggests a higher porosity degree in the films, which reduces their optical density. The enhancement of the nonlinear optical response of the films is attributed to two different origins. First, film glasses present a higher Ti atomic content, which increases their $\left|\chi^{(3)}\right|$ value due to the high hyperpolarizability of the $\mathrm{Ti}^{4+}$ cation. However, the observed compositional changes cannot explain alone the increase in $\left|\chi^{(3)}\right|$. The analysis of the structure of the glass network suggests that film glasses have a higher concentration of highly polarizable NBO, which contribute to enhance the nonlinear response of the films.

\section{ACKNOWLEDGMENTS}

The authors acknowledge the financial support of the Spanish Ministry of Education and Science (Project Nos.
MAT2005-06508-C02-01 and TEC2005-00074/MIC). D. Munoz-Martin and H. Fernandez acknowledge grants from CSIC-JAE and Spanish Ministry of Education and ScienceFPU programmes, respectively.

${ }^{1}$ M. Yamane and Y. Asahara, Glasses for photonics (Cambridge University Press, Cambridge, 2000).

${ }^{2}$ K. Tanaka, J. Mater. Sci. Mater. Electron. 16, 633 (2005).

${ }^{3}$ E. M. Vogel, M. J. Weber, and D. M. Krol, Phys. Chem. Glasses 32, 231 (1991).

${ }^{4}$ R. A. H. El-Mallawany, Tellurite Glasses Handbook: Physical Properties and Data (CRC, Boca Raton, FL, 2002).

${ }^{5}$ M. E. Lines, J. Appl. Phys. 69, 6876 (1991).

${ }^{6}$ K. S. Bindra, H. T. Bookey, A. K. Kar, B. S. Wherrett, X. Liu, and A. Jha, Appl. Phys. Lett. 79, 1939 (2001).

${ }^{7}$ H. Nasu, T. Uchigaki, K. Kamiya, and H. Kanbara, Jpn. J. Appl. Phys. 31, 3899 (1992)

${ }^{8}$ L. Canioni, M.-O. Martin, B. Bousquet, and L. Sarger, Opt. Commun. 151, 241 (1998)

${ }^{9}$ L. Weng and S. N. B. Hodgson, J. Non-Cryst. Solids 297, 18 (2002).

${ }^{10}$ F. D'Amore, M. Di Giulio, S. M. Pietralunga, A. Zappettini, L. Nasi, V. Rigato, and M. Martinelli, J. Appl. Phys. 94, 1654 (2003).

${ }^{11}$ M. Martino, A. P. Caricato, M. Fernández, G. Leggieri, A. Jha, M. Ferrari, and M. Mattarelli, Thin Solid Films 433, 39 (2003).

${ }^{12}$ K. L. Saenger, Process. Adv. Mater. 2, 1 (1993).

${ }^{13}$ J. Gonzalo, H. Fernandez, J. Solis, D. Munoz-Martin, J. M. FernandezNavarro, C. N. Afonso, and J. L. G. Fierro, Appl. Phys. Lett. 90, 251907 (2007).

${ }^{14}$ D. Munoz-Martin, J. Gonzalo, J. M. Fernandez-Navarro, J. Siegel, and C. N. Afonso, Appl. Surf. Sci. 254, 1111 (2007).

${ }^{15}$ M. A. Villegas and J. M. Fernandez-Navarro, J. Eur. Ceram. Soc. 27, 2715 (2007).

${ }^{16}$ D. A. Shirley, Phys. Rev. B 5, 4709 (1972).

${ }^{17}$ R. L. Sutherland, Handbook of Nonlinear Optics (Dekker, New York, 1996).

${ }^{18}$ V. O. Sokolov, V. G. Plotnichenko, V. V. Koltashev, and E. M. Dianov, J. Non-Cryst. Solids 352, 5618 (2006).

${ }^{19}$ T. Sekiya, N. Mochida, A. Ohtsuka, and M. Tonokawa, J. Ceram. Soc. Jpn. 97, 1435 (1989).

${ }^{20}$ T. Sekiya, N. Mochida, A. Ohtsuka, and M. Tonokawa, J. Non-Cryst. Solids 144, 128 (1992).

${ }^{21}$ G. S. Murugan and Y. Ohishi, J. Non-Cryst. Solids 351, 364 (2005).

${ }^{22}$ J. C. Sabadel, P. Armand, D. Cachau-Herreillat, P. Baldeck, O. Doclot, A. Ibanez, and E. Philippot, J. Solid State Chem. 132, 411 (1997).

${ }^{23}$ T. Komatsu, H. Tawarayama, H. Mohri, and K. Matusita, J. Non-Cryst. Solids 135, 105 (1991).

${ }^{24}$ K. Fukumi, T. Kokubo, K. Kamiya, and S. Sakka, J. Non-Cryst. Solids 84, 100 (1986).

${ }^{25}$ S. Kim and T. Yoko, J. Am. Ceram. Soc. 78, 1061 (1995).

${ }^{26}$ M. E. Lines, Phys. Rev. B 43, 11978 (1991).

${ }^{27}$ E. M. Vogel, S. G. Kosinski, D. M. Krol, J. L. Jackel, S. R. Friberg, M. K. Oliver, and J. D. Powers, J. Non-Cryst. Solids 107, 244 (1989).

${ }^{28}$ E. Fargin, A. Berthereau, T. Cardinal, G. Le Flem, L. Ducasse, L. Canioni, P. Segonds, L. Sarger, and A. Ducasse, J. Non-Cryst. Solids 203, 96 (1996).

${ }^{29}$ V. Dimitrov and S. Sakka, J. Appl. Phys. 79, 1736 (1996).

${ }^{30}$ M. Ohring, Materials Science of Thin Films, 2nd ed. (Academic, New York 2002)

${ }^{31}$ D. E. Aspnes, Thin Solid Films 89, 249 (1982).

${ }^{32}$ R. Adair, L. L. Chase, and S. Payne, J. Opt. Soc. Am. B 4, 875 (1987).

${ }^{33}$ A. P. Mirgorodsky, M. Soulis, P. Thomas, T. Merle-Méjean, and M. Smirnov, Phys. Rev. B 73, 134206 (2006).

${ }^{34}$ O. Noguera, T. Merle-Méjean, A. P. Mirgorodsky, M. B. Smirnov, P. Thomas, and J. C. Champarnaud-Mesjard, J. Non-Cryst. Solids 330, 50 (2003). 